\title{
MEASURING AIRBORNE DUST AND MIST
}

\begin{abstract}
$\mathrm{A}^{\mathrm{N}}$ $\mathrm{N}$ international colloquium on this subject was arranged jointly by the Staubforschungsinstitut des Hauptverbandes der gewerblichen Berufsgenossenschaften, Bonn, and the Institut National de Recherche Chimique Appliquée, Paris; it was held on October 22-23 at Freiburg (Breisgau) and was attended by about 60 scientists and engineers. The programme was divided into three sections, the first of which was concerned with number and mass concentration and the distribution of particle size. Introductory speeches were made by Prof. A. Winkel (Bonn) and A. P. Avy (Paris).

Dr. F. Hartogensis (The Hague) discussed the influence of microscopic techniques on the number of particles counted and outlined a case for the use of a well-defined system to eliminate the considerable variations in the numbers of fine particles which were visible. There was little evidence on the liability of $0.5 \mu$ particles to cause silicosis, but there was no doubt that large numbers of much finer particles were found in silicotic lungs.

The error in mean dust concentration as a function of the number of measurements, based on a log-normal distribution, was evaluated by J. Juda (Warsaw) and could be applied to gaseous pollutants. He also spoke of a method of size analysis based on pressure measure. ments up to $3 \mathrm{~mm}$ water while particles were sedimenting in liquid. Size analysis by measuring the light scattered by individual particles, while airborne, was possible with apparatus described by J. Bricard and J. Pradel (Paris). A very uniform zone of light was traversed by a jet of aerosol particles which scattered light at $90^{\circ}$ into a microscope provided with a photomultiplier cell. An advantage of the apparatus was that the aerosol did not have to be diluted so that natural clouds of liquid particles could be measured; unexpectedly, fine liquid and solid particles had beon found in eity fogs which formed at 90 per cent relative humidity, the saturation deficiency being due to sulphur trioxide.
\end{abstract}

The use of membrane filters for mine dust was explained by H. D. Bauer (Bochum), and H. Gessner (Zurich) had a now sodimentation apparatus for size analysis by weighing fractions. A. Berner and O. Preining (Vienna) presented a new calibration of the 'Cascade Impactor' which they used for sampling atmospheric radioactivity, on account of its ability to handle a large volume of air. W. Stöber (Gottingen) explained a theory of the deposition of particles in a conical aerosol centrifuge.

The Stokes diameter of a particle, evaluated from its sedimentation rate, is smaller than the projected area diameter, which is assessed microscopically. New measurements of the dust penetrating a sedimentation chamber betwoon horizontal plates had been made, using a thermal precipitator, by W. Walkenhorst (Bochum), who demonstrated that the ratio varied from $1 / 1 \cdot 3$ to $1 / 2 \cdot 2$ for glass quartz, talc and coal, and exhibited a considerable scatter in any one sample of dust.

W. Matla (Heerlen) presented a number of correlations between the quartz content and ash of airborne dusts which showed that the easily dotermined ash value was a good indication of the amount of quartz in the dusts. Before laying down permissible limits of dust concentration, due consideration would have to be given to the standardization of measuring techniques which at present varied widely, although the principle of selective sampling for the elimination of the coarser airborne dust was generally accepted.

The second section, dealing with selective sampling, was opened by Dr. C. N. Davies (London), who showed how a curve of lung retention against particle size could be derived by considering all the available distributions of airborne dust, and of dust recovered post mortem from the lungs, which had been obtained by electron microscopy. The British 'Hexhlet' selective dust sampler worked on a simple falling velocity separation formula which did not represent selection in the human bronchi and bronchioles but could be redesigned to do so. A cyclone separator, described by $\mathrm{H}$. Breuer (Essen), collected much finer dust which resembled lung dust more closely.

M. Landwehr (Bochum) had used a pre-impinger to separate the coarse fraction of dust, while finer particles, below $5 \mu$, passed through and were collected in a scrubber or on a membrane filter.

Two papers dealt with practical aspects of the British 'Hexhlet'. R. J. Hamilton (Isleworth) explained how it had been found necessary to increase the separation of the selector plates to $1.2 \mathrm{~mm}$ to avoid pick-up of deposited particles and to reduce the width of the entry to prevent external wind effects. K. G. Schmidt (Bonn) was critical of the performance of the instrument and had been troubled by corrosion of the aluminium plates which carried over into the fine fraction of dust.

The final section covered recording dust apparatus and opened with a description by A. P. Avy and M. Benarie (Paris) of a novel device in which an aerosol was sucked through an orifice so that the particles struck a microphone and could be recorded. F. Hartogensis had found that a compensated photoelectric smoke recorder was of some use for measuring industrial dust and had also used a paper-stain recorder. M. Raillière (Paris) compared the principles of measuring sideways or forwards scattered light and E. Walter (Bonn) claimed that photoelectric recording of dust concentration was possible for con. centrations ranging from less than $1 \mathrm{mg} / \mathrm{m}^{3}$ to $30 \mathrm{mg} / \mathrm{m}^{3}$.

Messrs. Leitz (Wetzlar) announced a new Tyndallscope incorporating a photomultiplier which obtained its high voltage from a potted transistor flip-flop condenser circuit. This romarkably sensitive dust recorder retains the sensitivity and portability of the well-known visual instrument.

The recording of aerosol particles by the contact E.M.F. which is developed on impact has been developed by $R$. Prochazka (Vienna and Munich) and A. Schütz (Bonn). The former described a finished instrument in which wall collisions as the serosol passed through a tube were amplified; concentrations down to $0.5 \mathrm{mg} / \mathrm{m}^{3}$ at flows up to $60 \mathrm{~m}^{3} / \mathrm{h}$ and particles down to $0.2 \mu$ could be dealt with. The apparatus of Schütz operated on collisions with a metal wire and was even more sensitive; when the dust concentration fell below $0.1 \mathrm{mg} / \mathrm{m}^{3}$ fluctuations developed owing to the ion content of the atmosphere which depended on temperature and humidity. It could advantageously be preceded by a selection device when used for recording industrial dusts.

The use of Zeleny tubes to establish the size distribution of fine stmospheric particles marked with thorium $B$ was described by G. Madelaine, J. Bricard, J. Pradel and F. Billard (Fontenay-aux-Roses and Paris). Using this aerosol the efficiency of a filter paper was found to change from 99.997 per cent to 99.993 per cent when the particle size increased from $10^{-3}$ to $10^{-1} \mu$. W. Coenen (Bonn) deposited small ions on dust particles and recorded the dust by the ion current.

The meeting concluded with a paper by W. Neuwirth (Freiburg), who had investigated correlations of atmospheric pollution with prevailing weather conditions.

The papers which were presented at Freiburg will be published in Staub early in 1964 and another meeting will be held in Paris next autumn. $\quad$ C. N. Davies 